DOI:

10.1038/nri1962

\section{Variable neutrophils}

Neutrophils have always been classified as innate immune cells: cells that are the first to reach a site of inflammation and that recognize pathogens through invariant receptors. Now, new research shows that a subpopulation of mammalian neutrophils expresses a T-cell receptor (TCR)-based variable immunoreceptor, indicating that neutrophils might use both innate and adaptive immune mechanisms for pathogen recognition.

Immunohistochemical analysis of peripheral-blood neutrophils stained with antibodies specific for the $\alpha$-chain and $\beta$-chain of the TCR showed that $5-8 \%$ of circulating neutrophils express both of these subunits. A similar percentage of $\mathrm{CD} 16^{\text {hi }}$ neutrophils were shown to express TCR $\alpha$, using flow cytometry. Using electron microscopy, the authors showed that these subunits formed heterodimers and higher-order TCR complexes, similar to those seen at the surface of $\mathrm{T}$ cells. They also showed that neutrophils expressed the constant regions of TCR $\gamma$ and TCR $\delta$. Transcriptional profiling of the mRNA encoding the variable

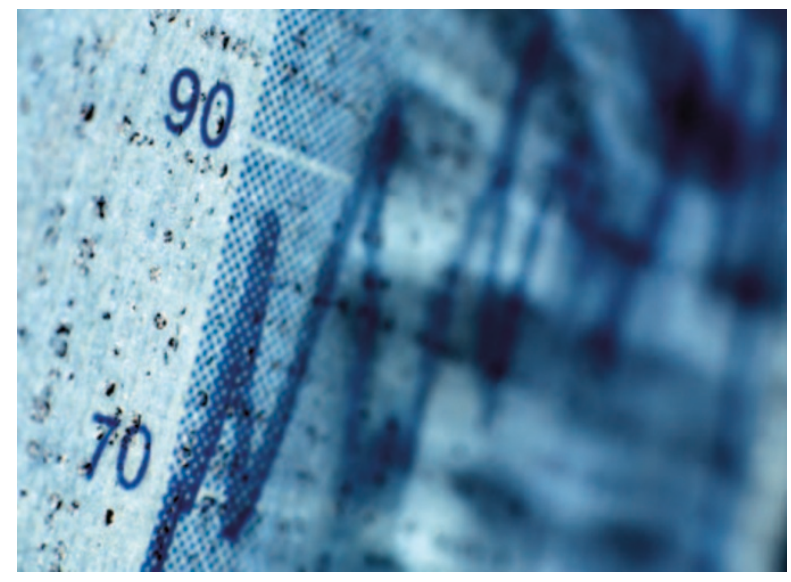

regions of TCR $\alpha$ and TCR $\beta$ showed great sequence variation, and the expression profile differed between individuals. Therefore, a subpopulation of neutrophils expresses TCRbased immunoreceptors that have complex variable regions and vary between individuals.

Interestingly, peripheral-blood neutrophils constitutively express recombination-activating gene 1 (RAG1) and RAG2 proteins, which initiate genomic $\mathrm{V}(\mathrm{D})$ J recombination, confirming that neutrophils express the specific proteins that are required for the generation of TCR diversity. Neutrophils also express components of the TCR-signalling complex, including the $\zeta$-chain of CD3, ZAP70 ( $\zeta$-chain-associated protein kinase of $70 \mathrm{kDa}$ ) and linker for activation of $\mathrm{T}$ cells (LAT).

The authors isolated neutrophils from a patient with X-linked severe combined immunodeficiency (a disorder that results in markedly reduced numbers of $\mathrm{T}$ cells) and from nude mice (which are devoid of $\mathrm{T}$ cells) and showed that these neutrophils still expressed the constant regions of TCR $\alpha$ and TCR $\beta$. Therefore, the expression of the TCR-based immunoreceptor by neutrophils is independent of TCR expression by $\mathrm{T}$ cells.

Expression of RAG1 and RAG2, as well as expression of the constant regions of the TCR chains, was shown to be regulated by granulocyte colony-stimulating factor, a factor that is important for the differentiation of neutrophil precursors. Stimulation of the neutrophil TCR-based immunoreceptor with CD3-specific antibody was found to increase expression of the antiapoptotic factor B-cell lymphoma $\mathrm{X}_{\mathrm{L}}$
(BCL- $\left.\mathrm{X}_{\mathrm{L}}\right)$, to protect neutrophils from apoptosis and to increase expression of the neutrophil-activating chemokine $\mathrm{CXC}$-chemokine ligand 8 (CXCL8; also known as IL-8).

Taken together, these results provide evidence that a subpopulation of mammalian neutrophils expresses a TCR-based variable immunoreceptor. Although the exact role of the variable immunoreceptor of these cells is not known, this unique observation by Puellmann and colleagues could lead to a greater understanding of the role of neutrophils and the TCR in the immune system.

Olive Leavy

\section{ORIGINAL RESEARCH PAPER}

Puellmann, K. et al. A variable immunoreceptor in a subpopulation of human neutrophils. Proc. Natl Acad. Sci. USA 103, 14441-14446 (2006)

FURTHER READING Nathan, C. Neutrophils and immunity: challenges and opportunities. Nature Rev. Immunol. 6, 173-182 (2006) 